\title{
Transferência de renda e segurança alimentar no Brasil: análise dos dados nacionais ${ }^{1}$
}

\section{Cash transference and food insecurity in Brazil: analysis of national data}

\author{
Ana Maria SEGALL-CORRÊA ${ }^{2}$ \\ Leticia MARIN-LEON ${ }^{2}$ \\ Hugo HELITO ${ }^{3}$ \\ Rafael PÉREZ-ESCAM ILLA ${ }^{4}$ \\ Leonor Maria Pacheco SANTOS 5 \\ Rômulo PAES-SOUSA ${ }^{6}$
}

\section{RE S U M O}

\section{Objetivo}

O objetivo deste trabalho foi analisar, na Pesquisa Nacional por Amostra de Domicílios 2004, a hipótese de que a participação em programas governamentais de Transferência de Renda está associada à situação de segurança alimentar no domicílio.

\section{Métodos}

Utilizaram-se dados secundários da Pesquisa Nacional por Amostra de Domicílios com entrevistas em 112716 domicílios. Incluíram-se neste trabalho os domicílios particulares permanentes e improvisados, em que as informações sobre segurança alimentar foram fornecidas por um morador, limitando-se ainda àqueles com rendimento domiciliar per capita de até um salário-mínimo, representando isto 51,2\% dos domicílios da amostra. Foram elaborados três modelos de estimativas de transferência de renda com resultados semelhantes; escolheu-se o Modelo 3 por resultar em menor probabilidade de superestimar efeitos. Para estimar o efeito da transferência de renda na prevalência de segurança alimentar, foram incluídos apenas os domicílios que recebiam transferência de renda correspondendo a 14,2\% do total de domicílios entrevistados. A associação entre segurança alimentar e transferência de renda, controlando por outras variáveis independentes, foi estimada mediante modelos de regressão logística, método stepwise, para cada uma das três faixas de rendimento domiciliar per capita.

\footnotetext{
${ }^{1}$ Artigo elaborado a partir de "Análise secundária de dados da Pesquisa Nacional por Amostra de Domicílios (PNAD)". Instituto Brasileiro de Geografia e Estatística. Rio de Janeiro; 2004.

2 Universidade Estadual de Campinas, Faculdade de Ciências Médicas, Departamento de Medicina Preventiva e Social. Caixa Postal 6111, Cidade Universitária, 13084-970, Campinas, SP, Brasil. Correspondência para/Correspondence to: A. SEGALL-CORRÊA. E-mail: <segall@fcm.unicamp.br>.

3 Universidade Estadual de Campinas, Faculdade de Ciências Médicas, Departamento de Medicina Preventiva e Social, Grupo de Pesquisa em Segurança Alimentar. Campinas, SP, Brasil.

4 University of Connecticut, NIH EXPORT Center of Excellence for Eliminating Health Disparities Among Latinos. Storrs CT, USA.

5 Ministério de Desenvolvimento Social e Combate à Fome, Secretaria de Avaliação e Gestão da Informação. Brasília, DF, Brasil.

6 Pontifícia Universidade Católica de Minas Gerais, Programa de Pós-Graduação em Ciências Sociais. Belo Horizonte, MG, Brasil.
} 


\section{Resultados}

Os programas de transferência de renda considerados neste estudo apresentaram um valor médio de benefícios de $\mathrm{R} \$ 81,68$ por domicílio. A regressão logística múltipla mostrou aumento em torno de 8,0\% na chance de segurança alimentar, para cada 10 reais de acréscimo nos valores das transferências. As condições de: residência em área rural, pessoa de referência do sexo masculino e de raça/cor branca também apresentaram associação positiva com segurança alimentar.

\section{Conclusão}

Os resultados confirmam a hipótese do estudo, indicando associação positiva da transferência de renda sobre a segurança alimentar, independentemente do efeito de outras condições explicativas.

Termos de indexação: Inquérito domiciliar. Pobreza. Política social. Segurança alimentar e nutricional.

\section{A B S T R A C T}

\section{Objective}

The 2004 National Household Survey Data was analyzed to test the hypothesis that cash transference from government social programs is associated with household food security.

\section{Methods}

Secondary data were used from the National Household Sample Survey which interview ed residents of 112,716 households. The present analysis included permanent and temporary private households, where food security items were informed by a resident of the household, restricting the collection of data to households with per capita monthly income of up to 1 minimum wage, representing $51.2 \%$ of the sample. Three models for estimating the amount of cash transference were developed; model 3 was chosen because it was less likely to overestimate effects. To analyze how cash transference affected the prevalence of food security, only households that received cash transference were included in the analysis, representing $14.2 \%$ of the interviewed households. The association between food security and cash transference, controlled for other independent variables, was estimated using a logistic regression model with stepwise method for each of the three income strata.

\section{Results}

The mean per capita cash transference was of $\mathrm{R} \$ 81.68$ per family. Multiple logistic regression showed an increased chance of food security of $8 \%$ per each $\mathrm{R} \$ 10.00$ contributed by social programs. Rural residence, male head of household, and Caucasian head of household were factors that also presented a higher chance of food security.

\section{Conclusion}

The hypothesis was confirmed, showing a positive association between cash transfer and household food security, regardless of the effects of other explanatory factors.

Indexing terms: Household survey. Proverty. Public policy. Food security.

\section{N T R O D U ÇÃ O}

0 acesso à alimentação em quantidade e qualidade adequadas, garantido por meios socialmente aceitáveis e de forma permanente, é considerado um direito humano básico. Esse acesso pode ser medido por uma escala que identifica a condição de segurança ou insegurança alimentar no domicílio e quantifica a magnitude destes fenômenos nas populações expostas a condições que limitam o direito humano à alimentação adequada.
A insegurança alimentar e a fome constituem uma dimensão da pobreza e da desigualdade social. Nos últimos anos, diversos autores têm apontado para uma queda da desigualdade social no Brasil. Ferreira et al. ${ }^{1}$, ao analisarem a desigualdade no período de 1981 a 2004, destacam a queda persistente da desigualdade a partir de 1993, sendo que $7,0 \%$ dessa queda ocorreu no período de 2001 a 2004, atingindo seu menor nível. Kakwani et al. ${ }^{2}$ observaram que, apesar de a renda per capita média ter diminuído, houve um consistente declínio da pobreza. Hoffmann ${ }^{3}$ 
aponta que, no período 1997 a 2004, enquanto a renda média real da população caiu 5,5\% , a renda média dos $40,0 \%$ mais pobres cresceu $11,4 \%$. Rocha ${ }^{4}$ descreve que entre 2003 e 2004 houve uma diminuição no número dos pobres em 2,4 milhões de pessoas, sendo que os indigentes diminuíram em dois milhões. Entretanto, a proporção de pobres nas áreas metropolitanas diminuiu menos que na área rural ${ }^{5}$.

É pertinente supor que o aumento do rendimento médio dos mais pobres tenha tido impacto nos níveis de segurança alimentar. A Pesquisa Nacional por Amostra de Domicílios (PNAD), de 2004, em seu suplemento de Segurança Alimentar ${ }^{6}$, mostrou que a proporção de insegurança alimentar grave diminui progressivamente à medida em que aumentam os rendimentos médios, mesmo em população de baixa renda. Assim, a proporção de insegurança alimentar moderada ou grave, em 2004, era de $61,2 \%$ na faixa de rendimento de até $1 / 4$ de saláriomínimo (SM) per capita, passando a 37,2\% na de mais de $1 / 4$ a $1 / 2$ e de $19 \%$ na faixa de mais de $1 / 2$ a 1 SM per capita.

Pesquisadores têm estudado a contribuição das Transferências de Renda (TR) para a queda da desigualdade e a diminuição da pobreza. Kakwani et al. ${ }^{2}$ destacam a criação do Fundo de Erradicação da Pobreza em 2000 e a progressiva inclusão, nos programas sociais, das famílias dos municípios de menor Índice de Desenvolvimento Humano (IDH). Paes de Barros et al. ${ }^{7}$ atribuem $20,0 \%$ da diminuição da desigualdade aos Programas de Transferência de Renda. Soares et al. ${ }^{8}$ atribuem $7,0 \%$ da queda da desigualdade de renda, medida pelo Índice de Gini, ao Benefício Assistencial de Prestação Continuada (BPC) e 21,0\% ao Programa Bolsa-Família. Esses achados são consistentes com os de Hoffmann ${ }^{3}$ que, no período 2002-2004, atribui 31,4\% da diminuição da desigualdade de renda ao aumento da cobertura dos benefícios de TR. No Nordeste, no mesmo período, esse decréscimo atingiu $86,9 \%$, visto que foi maior a cobertura da população elegível nessa região ${ }^{3}$.
As Políticas de transferência de renda podem ter papel relevante na melhoria das condições sociais da população, especialmente entre aqueles em situação de extrema pobreza. Sendo a renda monetária um preditor, ainda que não o único, da Segurança Alimentar (SA), é de supor que os ganhos em rendimento tenham, também, contribuído para a redução da insegurança alimentar e da fome.

Em uma amostra aleatória de 3 mil famílias beneficiárias do Programa Bolsa-Família, observouse que $94,2 \%$ das crianças estudadas realizavam três ou mais refeições ao dia. Contudo, na mesma investigação, foi constatado que 45,2\% acusavam insuficiência no acesso aos alimentos para nutrição infantil em, pelo menos, uma refeição no último mês. O Nordeste apresentou a mais alta proporção para este indicador negativo de acesso: $55,4 \%{ }^{9}$.

No período de realização da PNAD 2004, conviviam com o Bolsa-Família, criado em janeiro de 2004 (Lei no 10836) para unificar a gestão e a execução dos programas sociais de transferência de renda, o Bolsa-Escola, o Bolsa-Alimentação, o Auxílio-Gás, o Programa de Erradicação do Trabalho Infantil (PETI) e o Benefício Assistencial de Prestação Continuada (BPC). 0 objetivo principal do Bolsa-Família era a ampliação da cobertura da população mais vulnerável à insegurança alimentar, além da regularização dos benefícios sociais.

M esmo considerando que, nos últimos anos, tem ocorrido uma diminuição no custo relativo dos alimentos ${ }^{10}$, existe uma parcela da população com dificuldades no dia-a-dia para atingir o requerimento nutricional mínimo para atendimento às suas necessidades e de sua família ${ }^{6}$. Visando a entender esse processo nos grupos populacionais mais vulneráveis, este trabalho analisa, na PNAD 2004, a hipótese de que a participação em programas governamentais de transferência de renda está associada à situação de segurança alimentar no domicílio. 


\section{M ÉTO D O S}

Trata-se de estudo transversal utilizando o banco de dados da PNAD 20046. Do total de 139157 domicílios integrantes da amostra, foram realizadas entrevistas em 112716 domicílios. Na presente análise foram incluídos os domicílios particulares permanentes e improvisados, excluindo-se, portanto, as moradias coletivas. Optou-se ainda por excluir aqueles domicílios cujas informações sobre segurança alimentar tivessem sido fornecidas por não moradores do domicílio. Ao todo foram excluídos nesta fase $3,7 \%$ dos domicílios entrevistados.

No questionário da PNAD havia registro, mediante resposta Sim/Não, se, no mês de referência, algum morador do domicílio recebia dinheiro dos programas sociais: Auxílio-Gás, BolsaFamília, Cartão-Alimentação, Bolsa-Alimentação, Benefício Assistencial de Prestação Continuada (BPC), Bolsa-Escola e Programa de Erradicação do Trabalho Infantil (PETI). A pesquisa questionava também o número de moradores beneficiários dos programas Bolsa-Alimentação, BPC, Bolsa-Escola e PETI. É importante referir as limitações relativas aos registros nesta PNAD de algumas das características dos programas oficiais federais, recebidos por moradores do domicílio. No caso do Programa Bolsa-Família, o domicílio poderia receber o valor do benefício variável de acordo com a composição demográfica da família. Contudo, esse aspecto não foi registrado, como também não o foi o item "outros programas", que se refere, em muitos casos, aos programas estaduais e municipais de transferência de renda.

Na PNAD o rendimento domiciliar per capita foi composto por: salários e remunerações de trabalhadores formais, trabalhadores por conta própria e empregadores, aposentadorias e pensões pagas pelo Instituto Nacional de Seguridade Social, Governo Federal e entidades de previdência particular. Não havia informação em separado dos valores recebidos por meio de programas sociais de transferência de renda. Estes eram registrados juntamente com outras doações e outros rendimentos em uma variável denominada: juros de caderneta de poupança e de outras aplicações, dividendos e outros rendimentos que recebia normalmente no mês de referência, que, na presente análise, passa a ser denominada " juros" . Nesta variável estão incluídos, portanto, os valores de benefícios de transferência de renda recebidos. Como, para a análise proposta, requeria-se estimar os valores das transferências de rendimentos dos programas, fez-se necessário incluir apenas os domicílios com baixos rendimentos, dado que estes teriam baixa probabilidade de possuir aplicações financeiras, sendo possível assumir, por esta razão, que os valores observados para estes domicílios, na variável “juros” , referiam-se apenas aos valores dos benefícios sociais.

Os benefícios incluídos no levantamento da PNAD 2004 foram todos os existentes nesse ano, e que iam do beneficio de menor valor, o auxílio-gás ( $\mathrm{R} \$ 15,00$ a cada dois meses) ao de maior monta, o BPC, que corresponde a um salário-mínimo (SM) por idoso ou portador de deficiência.

Frente às limitações impostas pelas características dos registros, optou-se por analisar alternativas de modelos para as estimativas de valor domiciliar total das diversas modalidades de transferência de renda. A partir dessas estimativas, procedeu-se à análise do possível efeito do valor da transferência de renda sobre os padrões de segurança alimentar da população, limitando-se ainda essa avaliação, como já referido, aos domicílios com rendimento mensal per capita de até um salário-mínimo.

Foram definidos como domicílios beneficiados por TR aqueles em que, pelo menos, um morador era beneficiário de qualquer um dos programas, e delineados três modelos de estimativas do valor dessa transferência. As estimativas foram realizadas a partir das variáveis de TR descritas anteriormente e disponíveis no arquivo de "Domićlios" dos micro-dados da PNAD e da variável "Juros", no arquivo de "Pessoas" . Considerando-se que a variável rendimento mensal domiciliar é composta pela soma dos rendimentos 
individuais dos moradores do domicílio e que o valor monetário das TR era incorporado ao valor do rendimento domiciliar, foi necessário, para as análises propostas, excluir do rendimento mensal domiciliar, os valores dessas transferências. $\mathrm{Na}$ seqüência foi calculado o montante per capita, tanto da TR, como do rendimento mensal domiciliar. Para fins de apresentação deste texto, utiliza-se o valor em categorias de salários-mínimos, sendo o valor do SM de $\mathrm{R} \$ 260,00$ no período de referência da PNAD 2004.

\section{Método para estimativa do valor rece- bido pelos domicílios beneficiários dos programas federais de transferência de renda}

Nos procedimentos de estimativas dos valores das transferências de renda dos programas federais nos domicílios com até um salário-mínimo de rendimento domiciliar per capita, foram elaborados três modelos a partir de: (a) informações disponíveis na PNAD; (b) informações possíveis de serem inferidas pelos critérios dos programas; (c) pela freqüência de determinados valores contidos na variável "Juros" no banco de dados, considerados como sendo pertinentes ao recebimento de programas de transferência de renda do Governo Federal.

Dessa forma, os três modelos propostos utilizaram o valor monetário informado na variável "Juros" do banco de dados de "pessoas". Procedeu-se, em cada domicílio, à soma dos valores de todos os moradores para os quais foi referido algum benefício registrado como componente da variável "Juros", criando-se a variável "Juros Domiciliar".

\section{Modelo 1}

O primeiro modelo (M 1) partiu do princípio de que foi correta a declaração de recebimento de benefícios, bem como seus valores, quando este estava disponível no banco de dados. 0 modelo foi composto, portanto, por variáveis obtidas diretamente do banco de dados da PNAD e por outras, cujo valor foi estimado, sempre que seu registro estava presente, ou em situação em que o número de pessoas beneficiárias não era referido. 0 campo da PNAD ocorreu em um período de transição dos antigos programas de transferência de renda para o Bolsa-Família (Bolsa-Escola, Bolsa-Alimentação, Auxílio-Gás e Cartão-Alimentação).

Obteve-se informação completa e direta do banco da PNAD para os seguintes programas:

1. Cartão-Alimentação, que concedia $\mathrm{R} \$ 50,00$ por família;

2. Benefício Assistencial de Prestação Continuada (BPC), que concedia $\mathrm{R} \$ 260,00$ por pessoa, podendo chegar a dois beneficiários idosos não havendo cumulatividade para os portadores de deficiência;

3. Programa de Erradicação do Trabalho Infantil (PETI), quando constava a informação do número de membros que o recebia; o seu valor era considerado de acordo com os critérios do programa, que estipulava $R \$ 25,00$, para moradores de área rural e $\mathrm{R} \$ 40,00$ para moradores da área urbana.

4. Bolsa-Alimentação, que concedia $\mathrm{R} \$ 15,00$ por pessoa, até o máximo de três beneficiários. No Bolsa-Escola, de modo similar, considerou-se $\mathrm{R} \$ 15,00$ por estudante, até 0 máximo de três por família.

Foram estimados os valores para as varáveis com informação incompleta, utilizando-se os critérios a seguir. Relativamente ao Auxílio-Gás foi necessário estimar o valor mensal, porque este foi informado de forma ambígua, ora como $R \$ 15,00$, ora como $R \$ 7,00$ para o mês de referência tendo em vista que o estabelecido pelo Programa era de $\mathrm{R} \$ 15,00$ a cada dois meses. Quanto ao Programa Bolsa-Família, na PNAD, havia a identificação do domicílio no qual algum morador recebia esse benefício. Este programa concede dois tipos de benefício, que em 2004 possuíam os seguintes valores: um básico, de $\mathrm{R} \$ 50,00$ e um variável, de $\mathrm{R} \$ 15,00$. 0 benefício 
variável poderia ser concedido à família que possuísse até três membros nas seguintes condições: criança com idade inferior a 16 anos, gestante e mulher em condição de amamentação. As famílias que possuíam renda per capita inferior a $R \$ 50,00$ eram elegíveis para ambos os benefícios. Já as famílias possuidoras de renda per capita variando entre $R \$ 50,00$ e $R \$ 100,00$ eram elegíveis somente para o benéfico variável. As dificuldades nas estimativas referem-se ao fato de que, na PNAD, havia a identificação apenas do domicílio que recebia a transferência, sem, entretanto, registrar a composição familiar considerada para efeito de recebimento do benefício, nem quais recebiam o benefício básico.

A partir das considerações acima, procedeu-se às estimativas de valor mensal de transferência de renda da seguinte maneira. Definiuse inicialmente uma variável "modelo 1" (M1) que era formada pela soma dos valores correspondentes às informações obtidas diretamente do questionário da PNAD. Somou-se R $\$ 15,00$ para todos os que recebiam Auxílio-Gás e, também, $\mathrm{R} \$ 15,00$ para todos os que recebiam o benefício Bolsa-Família. Criou-se uma variável chamada "Resto M 1", subtraindo o valor obtido da variável M 1 (informação direta) do montante, para aquele domicílio, observado na variável "Juros Domiciliar": (Resto_M 1=Juros Domiciliar-M1).

Se o "Resto_M1" fosse igual a oito, este era considerado relativo ao benefício Auxilio-Gás cujo valor seria de $R \$ 7,00$ e a diferença subtraída da variável M1, para a devida correção. $\mathrm{Na}$ situação em que a variável "Resto_M1" fosse 0 montante possível do benefício Bolsa-Família, este valor era atribuído à variável M 1.

Se, após as atribuições de valores possíveis à variável $M 1$, ainda sobrasse algum valor na variável "Resto_M1", este retornava à variável "Juros Domiciliar".

\section{Crítica ao método}

Desconsiderou-se a possibilidade de ter havido erro na referência ao recebimento dos benefícios e do valor da transferência de rendimentos ter sido subestimado, dado que, em várias situações, restou "saldo positivo" na variável "Resto_M1".

\section{Modelo 2}

O segundo modelo (M 2) partiu do pressuposto de que o valor recebido de transferência não poderia ultrapassar aquele referido na variável "Juros Domiciliar". Utilizaram-se nestes procedimentos de estimativa, as variáveis de declaração de recebimento de programa de TR, mesmo considerando a possibilidade de erro nesta declaração.

Para sua operacionalização, considerou-se apenas uma família por domicílio, mesmo conhecendo a probabilidade de haver cerca de $5 \%$ dos domicílios com mais de uma família. Com os critérios de seleção usados neste trabalho, este percentual subiu a 13\% , na área urbana, e a $9 \%$ na área rural.

O valor de cada TR, iniciando-se com 0 BPC, foi sucessivamente sendo retirado da variável "Juros Domiciliar", somente enquanto existia algum montante nela referido.

\section{Crítica ao método}

Por este método podem ser desconsideradas informações de domicílios com declaração de recebimento de algum programa de transferência de renda e ainda, há a possibilidade de que sobre dinheiro na variável "Juros". Este método tende a subestimar o valor da transferência de renda e, portanto a superestimar seus efeitos sobre a condição de segurança alimentar da família.

\section{Modelo 3}

No terceiro modelo (M3) a informação de recebimento de transferência de renda no arquivo domicílios foi utilizada apenas para estimar o número total de beneficiários. Os valores das 
transferências de renda foram estimados, exclusivamente, a partir da variável "Juros Domiciliar". Foi considerado domicílio beneficiário todo aquele em que algum morador estivesse inscrito em, pelo menos, um programa oficial de TR.

Esta estimativa foi operacionalizada calculando-se o número de domicílios com programa de transferência de renda a partir da declaração de recebimento de pelo menos um dos benefícios. Seus valores foram considerados como aqueles constantes da variável "Juros Domiciliar", devendo ser ressaltado que a sub-amostra analisada era limitada, por definição prévia, aos domicílios com renda mensal per capita de, no máximo, um salário-mínimo.

\section{Crítica ao método}

Os procedimentos desse método desconsideram qualquer informação de declaração de valor de recebimento de benefício. 0 erro pode estar em superestimar o valor das transferências, uma vez que podem estar incluídos, nesta variável, outros rendimentos.

Ao final deste processo de estimativas os três modelos foram testados, chegando-se a valores muito próximos entre eles, em termos do número de beneficiários em cada faixa de valor recebido (Tabela 1 ).

Embora, em teoria, o modelo 3 possa superestimar o valor das transferências observou- se que, quando comparado aos modelos 1 e 2 , seus valores são mais conservadores para as faixas de maior rendimento, "R\$100,00-200,00" e “maior que $\mathrm{R} \$ 200,00$ ". Também foi observado que a proporção de domicílios na faixa "menor que $\mathrm{R} \$ 25,00$ per capita" foi discretamente maior no modelo M2. Logo, nesta faixa, o modelo M2 tende a subestimar o valor da TR, superestimando seus efeitos (Tabela 1). Essas razões levaram à escolha do modelo M 3 para as análises de segurança alimentar e transferência de renda.

\section{Método de análise da segurança alimentar considerando o Modelo 3 e variáveis preditoras}

Diante do objetivo deste estudo de estimar o efeito dos benefícios de transferência na prevalência de segurança/insegurança alimentar, segundo as categorias de rendimento domiciliar, incluiram-se na análise apenas os domicílios particulares que recebiam alguma modalidade de TR. Como o critério para escolha do informante do módulo de segurança alimentar, no estudo de validação do instrumento usado para diagnóstico da Segurança Alimentar/Insegurança Alimentar, foi ser um membro da família, informado sobre a disponibilidade de alimentose as opções familiares relativas à alimentação, no presente estudo optouse pela exclusão da análise de domicílios cujo informante tivesse sido um "Não Morador". No cálculo da renda domiciliar, para efeito de inclusão

Tabela 1. Distribuição populacional segundo valor de transferência de renda dos programas sociais em cada modelo de estimação. Brasil, 2004.

\begin{tabular}{lrrrrrr}
\hline Valor transferido per capita $(\mathrm{R} \$)^{*}$ & Modelo 1 & \multicolumn{1}{c}{$\%$} & Modelo 2 & \multicolumn{1}{c}{$\%$} & Modelo 3 & $\%$ \\
\hline$<25$ & 6501184 & 87,9 & 6237981 & 88,9 & 6314375 & 87,6 \\
$25-50$ & 291052 & 3,9 & 235883 & 3,4 & 395606 & 5,5 \\
$50-75$ & 241610 & 3,3 & 232286 & 3,3 & 252295 & 3,5 \\
$75-100$ & 135033 & 1,8 & 128546 & 1,8 & 134818 & 1,9 \\
$100-200$ & 139314 & 1,9 & 115078 & 1,6 & 98233 & 1,4 \\
$>200$ & 90271 & 1,2 & 67114 & 1,0 & 13778 & 0,2 \\
\hline Total & 7398464 & 100,0 & 7016888 & 100,0 & 7209105 & 100,0 \\
\hline
\end{tabular}

*valores em reais de transferência de renda estimados, pelos autores, a partir de informações coletadas na Pesquisa Nacional por Amostra de Domicílio, 2004. 
no estudo, não foram considerados os rendimentos decorrentes dos programas de TR. A análise considerou apenas os domicílios que declararam rendimento mensal domiciliar per capita de até um salário-mínimo; nesta situação, ainda sem exclusão dos valores de transferência de renda estimados pelos modelos, esta categoria representa $51,2 \%$ dos domicílios entrevistados. Dentre estes, $28,2 \%$ foram identificados como possuidores de algum tipo de TR. Quando combinados os atributos faixa salarial e recebimento de TR, os domicílios incluídos na PNAD 2004, correspondiam ao $14,2 \%$ do total das entrevistas realizadas.

A variável dependente, segurança/insegurança alimentar, para a finalidade desta análise, foi agrupada em duas categorias opostas, segurança alimentar ou insegurança leve e insegurança moderada ou grave. 0 valor per capita da transferência de renda em reais foi analisado como variável contínua.

Estudos anteriores, e a análise descritiva prévia dos dados da PNAD 20046 , apontam associação de diversas condições dos domicílios e indivíduos com a segurança/insegurança alimentar. Inicialmente, destacam-se as variáveis que tradicionalmente evidenciam desigualdades, como rendimento mensal domiciliar per capita e escolaridade da pessoa de referência do domicílio. A seguir, destacam-se as variáveis "sexo" e raça/cor da pessoa de referência. Foram, ainda, incluídas na análise de regressão múltipla, algumas características da composição domiciliar, tais como, existência de, pelo menos, um morador menor de 18 anos e de domicílios numerosos (com seis ou mais moradores). Todas as variáveis independentes que se mostraram, na análise univariada, significantemente associadas $(p<0,20)$ com insegurança alimentar moderada ou grave foram incluídas em um modelo multivariado em formato dicotômico, ou seja, característica presente/ausente ou em duas categorias para as variáveis contínuas, quando aplicável. Considerando que a satisfação das necessidades de alimentação está fortemente associada ao poder aquisitivo do grupo familiar, optou-se por delinear um modelo de regressão logística, método stepwise, para cada faixa de rendimento mensal domiciliar per capita, menos de $1 / 4$ SM, de $1 / 4$ a $1 / 2$ SM e de mais de $1 / 2$ a $1 \mathrm{SM}$. Desses rendimentos foram excluídos os valores de transferência de renda observados ou, estimados conforme o Modelo 3.

\section{RESULTA DOS}

A Tabela 2 descreve o perfil dos domicílios urbanos e rurais observados na PNAD 2004, nela estão cotejados os percentuais obtidos para domicílios de baixa renda beneficiários e não beneficiários de programas de transferência de renda.

As modalidades de transferência de renda consideradas neste estudo apresentaram um valor médio dos benefícios de $\mathrm{R} \$ 81,68$ por domicílio. Não é possível uma comparação direta com o valor médio praticado pelo Programa Bolsa-Família que, à época, transferia, em média, $\mathrm{R} \$ 68,13$ por família ${ }^{11}$, pelo fato de a PNAD se reportar a domicílios e o Bolsa-Família a famílias. Considerando as restrições para a inclusão dos domicílios nesta análise, os valores referem-se a sete milhões de domicílios permanentes não coletivos, tanto urbanos como rurais e, para os quais, a pessoa que prestou as informações era um residente.

$\mathrm{Na}$ Tabela 3, apresentam-se as variáveis, que integram os modelos finais de regressão logística para o País como um todo e segundo três estratos de rendimentos médios familiares per capita de até 1 salário-mínimo. As categorias de cada variável, apresentadas nesta Tabela, são aquelas associadas à maior chance de segurança alimentar ou insegurança alimentar leve, por exemplo, em cada uma das faixas de renda, os domicílios com pessoa de referência do sexo masculino têm maior chance de apresentar segurança alimentar ou insegurança alimentar leve do que naqueles domicílios cujas pessoas de referência são mulheres. 
Tabela 2. Proporção de características sócio-demográficas selecionadas, em domicílios com rendimento mensal per capita de até um salário-mínimo, segundo situação de residência e recebimento de Transferência de Renda (TR) estimada pelo modelo 3 (M3). Brasil, 2004.

\begin{tabular}{|c|c|c|c|c|c|}
\hline \multirow{2}{*}{ Variável } & \multirow{2}{*}{ Categoria } & \multicolumn{2}{|c|}{ Urbana ${ }^{a}$} & \multicolumn{2}{|c|}{ Rurala $^{\mathbf{a}}$} \\
\hline & & TR $(n=32414)$ & Com TR ( $n=11415)$ & Sem TR $(n=7518)$ & Com TR $(n=4690)$ \\
\hline \multirow[t]{3}{*}{ Rendimento per capita } & $<1 / 4$ & 9,1 & 22,9 & 18,7 & 38,0 \\
\hline & $1 / 4-1 / 2$ & 27,1 & 41,8 & 30,7 & 39,9 \\
\hline & $>1 / 2-1$ & 63,8 & 35,3 & 50,6 & 22,1 \\
\hline \multirow[t]{2}{*}{ Sexo da pessoa de referência } & Masculino & 70,2 & 70,1 & 86,1 & 88,3 \\
\hline & Feminino & 29,8 & 29,9 & 13,9 & 11,7 \\
\hline \multirow{2}{*}{$\begin{array}{l}\text { Anos de estudo da pessoa de } \\
\text { referência }\end{array}$} & $0 \leq 3$ anos & 28,7 & 40,3 & 52,9 & 61,4 \\
\hline & $>3$ anos & 71,3 & 59,7 & 47,1 & 38,6 \\
\hline \multirow{2}{*}{$\begin{array}{l}\text { Raça/cor da pessoa de referên- } \\
\text { cia }\end{array}$} & Preta/Parda & 54,8 & 66,8 & 61,0 & 66,9 \\
\hline & Branca & 45,2 & 33,2 & 39,0 & 33,1 \\
\hline \multirow[t]{2}{*}{ Tem morador $\leq 5$ anos } & Sim & 37,4 & 40,8 & 36,5 & 41,6 \\
\hline & Não & 62,6 & 59,2 & 63,5 & 58,4 \\
\hline \multirow[t]{2}{*}{ Tem morador $\leq 18$ anos } & Sim & 73,4 & 94,2 & 67,6 & 93,8 \\
\hline & Não & 26,6 & 5,8 & 32,4 & 6,2 \\
\hline \multirow[t]{2}{*}{ Tem morador $\geq 65$ anos } & Sim & 17,3 & 12,6 & 21,5 & 10,1 \\
\hline & Não & 82,7 & 87,4 & 78,5 & 89,9 \\
\hline \multirow[t]{2}{*}{ Número moradores } & $<6$ & 87,6 & 70,9 & 87,6 & 64,7 \\
\hline & $\geq 6$ & 12,4 & 29,1 & 12,4 & 35,3 \\
\hline \multirow[t]{2}{*}{ Número famílias } & 1 & 90,3 & 86,5 & 93,7 & 90,8 \\
\hline & $>1$ & 9,7 & 13,5 & 6,3 & 9,2 \\
\hline \multirow[t]{2}{*}{ Número pessoa/dormitório } & $\leq 2$ & 67,7 & 54,0 & 70,4 & 56,6 \\
\hline & $>2$ & 32,3 & 46,0 & 29,6 & 43,4 \\
\hline \multirow[t]{2}{*}{ Tem banheiro } & $\mathrm{Sim}$ & 97,2 & 94,0 & 73,0 & 63,0 \\
\hline & Não & 2,8 & 5,9 & 27,0 & 37,0 \\
\hline \multirow{2}{*}{$\begin{array}{l}\text { A pessoa de referência tem } \\
\text { emprego }\end{array}$} & Sim & 67,0 & 70,6 & 80,0 & 88,0 \\
\hline & Não & 33,0 & 29,4 & 20,0 & 12,0 \\
\hline \multirow[t]{2}{*}{ Composição familiar } & Casal c/ ou s/ filhos & 68,1 & 72,3 & 77,0 & 86,5 \\
\hline & Outras & 31,9 & 27,7 & 23,0 & 13,5 \\
\hline \multirow[t]{2}{*}{ Situação censitária } & Região metropolitana & a 66,2 & 78,8 & 93,7 & 96,9 \\
\hline & Outras & 33,8 & 21,2 & 6,3 & 3,1 \\
\hline
\end{tabular}

a 0 n de cada coluna refere-se ao número de domicílios entrevistados, mas a proporção foi calculada com a população expandida, considerando o desenho amostral.

Os resultados da análise de regressão logística múltipla mostraram que, em todas as faixas de rendimento familiar, houve aumento, em torno de $8 \%$ na chance de segurança alimentar na família, para cada 10 reais de acréscimo nos valores das transferências socais. Outras variáveis, tais como a situação de residência em área rural, pessoa de referência do sexo masculino, entrevistado de raça/cor branca, entre outras, apresentaram associação ainda mais forte com a segurança alimentar (Tabela 3 ), neste grupo populacional.

\section{I S C U S SÃ 0}

0 presente estudo fez inferências considerando o pressuposto de que em domicílios com rendimento domiciliar per capita de até um saláriomínimo, os valores declarados na variável juros de caderneta de poupança e de outras aplicações, dividendos e outros rendimentos que recebia normalmente no mês de referência ou, aqui denominada "Juros", referem-se às transferências de renda dos programas sociais. A hipótese se justifica uma vez que as famílias não teriam, neste 
Tabela 3. Condições preditoras de segurança alimentar/insegurança levea ${ }^{a}$, estimadas mediante modelo de regressão logística, considerando os domicílios que recebiam benefícios de transferência de renda. Brasil, 2004.

\begin{tabular}{|c|c|c|c|c|c|}
\hline \multirow{2}{*}{$\begin{array}{l}\text { Rendimento domiciliar } \\
\text { per capita } \\
<1 / 4 \text { salário-mínimo }(n=5040)\end{array}$} & \multirow{2}{*}{$\begin{array}{l}\text { Variável } \\
\text { Incremento de transferência de renda de } \mathrm{R} \$ 10\end{array}$} & \multirow{2}{*}{$\begin{array}{l}p \text { valor } \\
<0,001\end{array}$} & \multirow{2}{*}{$\frac{\text { OR }^{\mathrm{a}} \text { ajustado }}{1,08}$} & \multicolumn{2}{|c|}{ IC $95 \%$ b } \\
\hline & & & & 1,04 & 1,12 \\
\hline & Pessoa de referência do sexo masculino & $<0,001$ & 1,64 & 1,31 & 2,04 \\
\hline & Situação rural & $<0,001$ & 1,88 & 1,60 & 2,21 \\
\hline & Escolaridade da pessoa de referência $\geq 4$ anos & $<0,001$ & 1,43 & 1,22 & 1,68 \\
\hline & Raça/cor branca da pessoa de referência & $<0,001$ & 1,39 & 1,18 & 1,64 \\
\hline & Domicílios com até 5 moradores & $<0,001$ & 1,38 & 1,18 & 1,62 \\
\hline & Domicílio com pelo menos 1 morador $<18$ anos & 0,023 & 1,91 & 1,09 & 3.35 \\
\hline \multicolumn{6}{|c|}{$>1 / 4-1 / 2$ salário-mínimo $(n=6347)$} \\
\hline & Incremento de transferência de renda de $\mathrm{R} \$ 10$ & $<0,001$ & 1,08 & 1,05 & 1,11 \\
\hline & Pessoa de referência do sexo masculino & $<0,001$ & 1,47 & 1,28 & 1,67 \\
\hline & Situação Rural & $<0,001$ & 1,75 & 1,54 & 1,98 \\
\hline & Escolaridade da pessoa de referência $\geq 4$ anos & $<0,001$ & 1,25 & 1,11 & 1,40 \\
\hline & Raça/cor branca da pessoa de referência & $<0,001$ & 1,41 & 1,26 & 1,58 \\
\hline & Domicílios com até 5 moradores & 0,002 & 1,23 & 1,08 & 1,40 \\
\hline & Domicílio com pelo menos 1 morador <18anos & 0,031 & 1.30 & 1,02 & 1,65 \\
\hline & Região não metropolitana & 0,003 & 1.22 & 1.07 & 1.40 \\
\hline & Até 2 pessoas por dormitório & $<0,001$ & 1.33 & 1.18 & 1.49 \\
\hline \multicolumn{6}{|l|}{$>1 / 2-1$ salário-mínimo $(n=3730)$} \\
\hline & Incremento de transferência de renda de $\mathrm{R} \$ 10$ & $<0,001$ & 1,07 & 1,04 & 1,10 \\
\hline & Pessoa de referência do sexo masculino & $<0,001$ & 1,36 & 1,17 & 1,59 \\
\hline & Situação Rural & $<0,001$ & 1.63 & 1.37 & 1.94 \\
\hline & Escolaridade da pessoa de referência $\geq 4$ anos & $<0,001$ & 1,34 & 1,16 & 1,54 \\
\hline & Raça/cor branca da pessoa de referência & $<0,001$ & 1,48 & 1,29 & 1,69 \\
\hline & Domicílios com até 5 moradores & 0,077 & 1,17 & 0,98 & 1,39 \\
\hline & Domicílio com pelo menos 1 morador $<18$ anos & 0,053 & 1.25 & 1,00 & 1,58 \\
\hline & Região não metropolitana & $<0,001$ & 1.37 & 1.17 & 1.60 \\
\hline & Até 2 pessoas por dormitório & 0,055 & 1,16 & 0,99 & 1,36 \\
\hline
\end{tabular}

Variável dependente: segurança alimentar, com duas categorias, segurança alimentar/insegurança leve e insegurança. moderada/insegurança grave (categoria de referência). a Odds Ratio; ' ${ }^{\mathbf{b}}$ Intervalo de confiança.

limite de renda, recursos para aplicações financeiras. 0 método escolhido para a estimativa do valor de transferência de renda é um método conservador, no que se refere à modificação da chance de segurança alimentar associada ao aumento do valor das transferências, ou seja, é um método que subestima possíveis efeitos.

Deve ser lembrado que os domicílios que recebiam transferência de renda em 2004 correspondiam a uma parcela de cerca de $50 \%$ da população que atendia às condições definidas por lei como de potenciais beneficiários. Soares et al. ${ }^{8}$ apontam, no entanto, que a PNAD estima o número de beneficiários do Programa BolsaEscola quase $15 \%$ maior que o registrado pelo M inistério de Desenvolvimento Social e Combate à Fome ${ }^{11}$ e um número de beneficiários do BolsaFamília inferior à metade do registro oficial e que, em termos relativos, reproduz bem a distribuição regional e as características dos domicílios beneficiários.

O programa Bolsa-Família foi implantado em 2004, para unificar programas já existentes: Auxílio-Gás, Bolsa-Alimentação, Bolsa-Escola e Cartão-Alimentação. Por esta razão, quando questionados sobre o tipo de transferência de renda recebida, os informantes podem ter declarado ser beneficiários de programas não mais vigentes nos quais, anteriormente, haviam sido cadastrados. Esse viés de informação, entretanto, não afeta a estimativa do valor da TR, porque o método escoIhido emprega somente o valor existente na 
variável "Juros" e não considera a informação sobre o tipo de transferência de renda recebida.

Camargo ${ }^{12}$ crítica a Política Social atual, por ter privilegiado os idosos com valores de transferência maiores e descuidado das famílias com crianças. Nas análises de regressão logística, considerando a condição de segurança alimentar como variável dependente e o valor de TR como uma das variáveis preditoras, não foi observada associação estatisticamente significante entre as condições de ser família com moradores menores de cinco anos ou, família com idosos. Ou seja, controlando-se pelo efeito da renda, a composição familiar deixa de ter impacto sobre o padrão de segurança alimentar da família (dados não apresentados em tabelas). Entretanto, os domicílios com menores de cinco anos, apresentam valor médio de transferência de renda menor que os demais domicílios, sendo possível a hipótese de que outras características desses domicílios agregam condição protetora, contrapondo-se ao baixo rendimento domiciliar per capita.

A partir de informações de inquérito em domicílios mais pobres da cidade de São Paulo, Figueiredo et al. ${ }^{13}$ observaram que, em 2004, a cobertura dos programas de transferência de renda federais, estaduais ou municipais atingia $28 \%$ da população com menos de meio salário-mínimo per capita. Torres et al. ${ }^{14}$ descrevem que $63 \%$ das famílias que recebiam algum benefício de transferência de renda tinham renda familiar per capita de até meio salário-mínimo e 32\% entre meio e um salário-mínimo, portanto $95 \%$ estavam na categoria dos mais pobres. Nos domicílios entrevistados pela PNAD verificou-se proporção semelhante de beneficiários entre os mais pobres.

Os resultados da PNAD ${ }^{6}$ também mostraram situação de insegurança alimentar mais freqüente nos domicílios em que algum morador era beneficiário de programas de transferência de renda. Este é um resultado aparentemente paradoxal que pode estar relacionado a uma pior situação social desses domicílios, mesmo considerando uma mesma faixa de rendimento, o que teria favorecido também a inclusão nos programas.
Como se trata de estudo transversal, as análises de avaliação de efeito apresentam limitações, por outro lado os procedimentos adotados permitiram estimar o efeito da transferência de renda sobre a segurança alimentar ao realocar os domicílios em faixas de rendimento excluídos os valores de TR. 0 presente estudo aponta que 0 incremento no valor de transferência de renda aumenta a chance de segurança alimentar ou insegurança leve, isto é, afasta as famílias da insegurança moderada ou grave, que se constitui em uma expressão de maior pobreza. De fato, a análise econométrica dos dados de 15 mil famílias, incluídas no estudo de Avaliação de Impacto do Programa Bolsa-Família, aponta na mesma direção. Os diferenciais monetários entre o grupo de beneficiários do Bolsa-Família e o grupo comparação (ambos com renda per capita inferior a $\mathrm{R} \$ 50,00$ ) mostram que o primeiro grupo gasta de $\mathrm{R} \$ 388,22$ a $R \$ 588,01$ a mais com alimentação por ano ( $p$ valor $<0,01)^{15}$. Este fato confirma também as considerações de Paes de Barros ${ }^{16}$ quanto ao fato de que o aumento do Bolsa-Família seria uma forma eficiente para diminuir a pobreza e a desigualdade, pois esse beneficio atinge não apenas os assalariados do setor formal, mas do informal, e desempregados.

Domicílios chefiados por mulher apresentaram maior chance de insegurança moderada ou grave em todas as faixas estudadas, de rendimento domiciliar per capita, fato que, certamente, guarda relação com outras características que desfavorecem as famílias que têm mulheres como pessoa de referência e provedoras das condições de sobrevivência.

As estimativas de transferência de renda apresentadas e sua associação com o aumento da chance de segurança alimentar representam uma média para o País. Entretanto, Rocha ${ }^{5}$ observou variações do custo de vida, segundo grandes regiões, sendo este menor em área rural que na urbana e ainda mais elevado nas metrópoles. Assim, pode-se prever que o impacto relativo de um mesmo valor de transferência de renda será diferente conforme a realidade local. 
No entanto, limitam esse detalhamento, a baixa cobertura que os programas de transferência de renda tinham em 2004 e, ainda, o delineamento amostral da PNAD.

Soares et al. ${ }^{8}$ apontam que o BPC e 0 Programa Bolsa-Família são bem focalizados; $74 \%$ das famílias que receberam BPC estavam abaixo da linha de pobreza ( $1 \frac{1}{2}$ salário-mínimo per capita); esse valor foi de $80 \%$ para o Bolsa-Família.

No período 1995-2005, em 11 regiões metropolitanas, Torres et al. ${ }^{14}$ observaram que, enquanto a inflação medida pelo Índice Nacional de Preço ao Consumidor (INPC) foi de $147 \%$, a variação dos alimentos foi de $92 \%$, isto é, em relação à inflação, os preços médios dos alimentos caíram. A análise de regressão logística aqui apresentada aponta que residir em área metropolitana aumenta a chance de insegurança alimentar, apesar do custo da alimentação ter diminuído, isto é significativo nos domicílios com rendimento domiciliar per capita, superior a $1 / 4 \mathrm{de}$ salário-mínimo (excluída a transferência de renda). Nessas áreas urbanas o custo dos itens não alimentares, possivelmente, compete com outras necessidades básicas das famílias.

A situação de residência em meio rural favorece a chance de segurança alimentar, embora os valores de transferência de renda para estas famílias sejam inferiores. Possivelmente, tanto a produção para auto-consumo como gastos menores com outros itens básicos, tais como vestuário e transporte, sejam determinantes da maior segurança alimentar relativa na área rural, o que contrasta com a situação em áreas urbanas metropolitanas.

\section{O N C L U SÃ O}

A comparação entre os três métodos de estimativa de valores de transferência de renda de programas sociais mostrou-se consistente, permitindo, escolhido um deles, a análise de sua associação com os padrões de segurança alimentar.
Os resultados obtidos indicam associação positiva da transferência de renda, com a segurança alimentar, independentemente do efeito de outras condições. M ostram, ainda, que esta associação é mais expressiva entre aqueles domicílios de muito baixo rendimento (até $1 / 4$ salário-mínimo per capita) e aqueles da faixa superior a $1 / 2$ até um salário-mínimo per capita, após a exclusão dos valores de transferência estimados. Deve ser lembrado que, na escolha do método para obter esta estimativa, optou-se por aquele com maior chance de subestimar os efeitos da transferência de renda sobre a situação de segurança alimentar no domicílio, o que reforça os resultados da associação observada.

Os resultados das análises aqui apresentadas demonstram, de forma consistente, os efeitos dos programas sociais de transferência de renda na redução da insegurança alimentar, e podem constituir em informações de base relevantes para o acompanhamento e a avaliação das políticas públicas que lhes dão sustentação.

\section{COLABORADORES}

A concepção do tema é fruto de discussão coletiva. A.M. SEGALL-CORRÊA e L. MARIN-LEON elaboraram e redigiram o corpo deste artigo. H. HELITO, com a colaboração de R. PÉREZ-ESCAM ILLA e L. MARINLEON, formulou os modelos de estimativa do valor de transferência de renda e as análises estatísticas. L.M.P. SANTOS e R. PAES-SOUSA participaram da revisão de todas as versões do artigo, realizando importantes contribuições à versão final do manuscrito.

\section{RE FERÊ N C I A S}

1. Ferreira FHG, Leite PG, Litchfield JA, Ulyssea G. Ascensão e queda da desigualdade de renda no Brasil. Econômica. 2006; 8(1):147-69.

2. Kakwani N, Son H, Neri M. Estagnação econômica e crescimento pró-pobre. Conjuntura Econômica. 2006; (6):70-1.

3. Hoffmann R. Transferências de renda e a redução de desigualdade no Brasil e cinco regiões entre 1997 e 2004. Econômica. 2006; 8(1):113-39. 
4. Rocha S. Impacto sobre a pobreza dos novos programas federais de transferência de renda. Econ Contemp. 2005; 9(1):153-85.

5. Rocha S. Pobreza e indigência no Brasil: algumas evidências empíricas com base na PNAD 2004. Nova Econ. 2006; 16(2):3-29.

6. Instituto Brasileiro de Geografia e Estatística. Pesquisa Nacional por Amostra de Domicílios. PNAD Segurança Alimentar 2004. Rio de Janeiro: IBGE; 2006.

7. Paes de Barros R, Carvalho M, Franco S. Brasil está menos desigual. O Globo, n.30 de janeiro de 2006 [acesso 2007 abr 10]. Disponível em: <http://www. jornaldaciencia.org.br/Detalhe.jsp? id=34909>.

8. Soares FV, Soares S, Medeiros M, Osório RG. Programas de transferência de renda no Brasil: impactos sobre a desigualdade. Texto para Discussão no 1228. Brasília; outubro; 2006 JEL:D31 [acesso 2007 abr 10]. Disponível em: <http://www. anpec.org.br/encontro2006/artigos/A06A156. pdf $>$.

9. Silva MCM, Assis AMO, Santana MLP, Pinheiro SMC, Santos NS, Brito E. Programa Bolsa-Família e segurança alimentar das famílias beneficiárias: resultados para o Brasil e regiões. In: Brasil. Ministério do Desenvolvimento Social e Combate à Fome. Avaliação de políticas e programas do MDS: Resultados. Brasília: MDS; 2007. v.2:69-96.

10. Brasil, Instituto de Pesquisa Econômica Aplicada (IPEA) Nota Técnica sobre a recente queda da desigualdade de renda no Brasil. Nota Técnica número 9. Brasília, 30 de agosto de 2006 [acesso 2007 abr 10]. Disponível em: <http://www.ipea. gov.br/sites/000/2/publicacoes/notastecnicas/ notastecnicas9.pdf >
11. Brasil. Ministério do Desenvolvimento Social e Combate à Fome. Dados 2004 [acesso 2007 abr 10]. Disponível em: <http://www.mds.gov.br/sagi/ estudos-e-pesquisas/folders/folders-da-sagi/>.

12. Camargo JM. Política Social no Brasil: prioridades erradas, incentivos perversos. São Paulo em Perspectiva. 2004; 18(2):68-77.

13. Figueiredo AC, Torres HG, Bichir RM . A conjuntura social brasileira revisitada. Novos Est Cebrap. 2006; 75(julh):173-83.

14. Torres HG, Bichir RM, Carpim TP. Uma pobreza diferente? Mudanças no padrão de consumo da população de baixa renda. Novos Est Cebrap. 2006; 74(mar):17-22.

15. Oliveira AMHC, Andrade MV, Resende ACC, Rodrigues CG, Souza LR, Ribas RP. Primeiros resultados da análise da linha de base da pesquisa de Avaliação de Impacto do Programa BolsaFamília. In: Brasil. Ministério do Desenvolvimento Social e Combate à Fome. Avaliação de Políticas e Programas do MDS - Resultados. v. 2. Brasília: MDS; 2007. v.2; p.19-66. Resumo executivo [acesso 2007 nov 20]. Disponível em: <http://www. mds.gov.br/ institucional/secretarias/secretaria-de-avaliacao-egestao-da-informacao-sagi/pesquisas>.

16. Paes de Barros R. Falta uma verdadeira estratégia social. Entrevista de Sérgio Lamucci ao economista Ricardo Paes de Barros coordenador de avaliação de políticas públicas do Instituto de Pesquisa Econômica Aplicada (Ipea) IETS s/d [acesso 2007 23 jan]. Disponível em: <http:/www.iets.org.br/ article.php3?id_article $=464>$.

Recebido em:14/8/2007

Versão final reapresentada em: 9/5/2008

Aprovado em: 10/6/2008 
